\section{SWITCHLESS BIDIRECTIONAL AMPLIFIER FOR WIRELESS COMMUNICATION SYSTEMS}

\author{
A. Sevinc Aydinlik Bechteler and Thomas F. Bechteler \\ Department of Electrical and Electronics Engineering, Izmir Institute of \\ Technology, 35430 Izmir, Turkey
}

Received 26 December 2006

ABSTRACT: A bidirectional amplifier working at $2.4 \mathrm{GHz}$ is presented in this article. The presented amplifier is able to amplify two signals simultaneously. This, since commonly used radio frequency switches are replaced by circulators. The amplifier is designed on a single smallsized printed circuit board. First, the new amplifier circuit is proposed and characterized. Then, its advantages and design problems are analyzed. Finally, design instructions are setup in order to obtain a good performance of the switchless amplifier within the frequency band of interest. () 2007 Wiley Periodicals, Inc. Microwave Opt Technol Lett 49: 1888-1890, 2007; Published online in Wiley InterScience (www. interscience.wiley.com). DOI 10.1002/mop.22631

Key words: RF amplifiers; transceivers; wireless systems; circulator; full duplex

\section{INTRODUCTION}

In the recent past, wireless communication systems have been developed rapidly. Wireless systems are preferably designed as integrated one-chip solutions which are cheap and tiny. For some applications, however, add-on systems like amplifiers are required to improve the performance of existing wireless systems. Usually, the transmitter power or the receiver sensitivity has to be improved which both results in a farther communication range of a wireless system.

During the past, different concepts for bidirectional amplifiers were presented. With respect to functionality, bidirectional amplifiers can be divided into half-duplex and full-duplex systems. With half-duplex amplifiers only one signal at one time can be amplified, and full-duplex amplifiers amplify two signals simultaneously.

Most commercial bidirectional amplifiers use radio frequency (RF) switches which switch between two signal paths [1,2]. Their switching time can cause data loss at a high transfer rate. For instance, with a data rate of $54 \mathrm{Mbit} / \mathrm{s}$ the time duration for $1 \mathrm{bit}$ is $17.66 \mathrm{~ns}$. Switching times of commercial available bidirectional amplifiers can be as high as $2 \mathrm{~ms}$ [1], causing a loss of more than 100 bits. Another switching concept is presented in [3] and [4]. There, bidirectional amplifiers realized as monolithic microwave integrated circuits (MMIC) bias a transistor circuit in such a way that it amplifies either in the one or the other direction. Full-duplex bidirectional amplifiers are presented for instance in [5-8]. In [5] a transistorized-directional coupler and in [6] a microstriplinedirectional coupler is used to combine two amplifiers for fullduplex applications. However, a transistorized-directional coupler shows a high noise and can handle only low power, and microstripline-directional couplers become too big for some applications. In $[7,8]$ distributed amplifiers are used to obtain a bidirectional effect without switching. Those amplifiers show high noise and low power handling capacity.

In the following, a switchless bidirectional amplifier is discussed. It is compared against a bidirectional amplifier concept using RF switches as commonly used in commercial wireless communication systems.

\section{THE SWITCHLESS BIDIRECTIONAL AMPLIFIER}

\subsection{First Design and Problems}

In Figure 1 the schematic diagram of a switchless bidirectional amplifier for $2.4 \mathrm{GHz}$ using circulators is depicted. Signal 1 is directed from port A to port B of circulator 1, thereby being attenuated due to the circulators insertion loss. It is then amplified by amplifier 1 and finally goes through circulator 2 . The same applies for signal 2 in the reverse direction. Compared with the insertion loss of RF switches, the insertion loss of circulators is low. A typical insertion loss value for circulators is $0.2 \mathrm{~dB}$ at 2.4 $\mathrm{GHz}$, whereas RF switches show values of about 1-dB insertion loss. Lower insertion losses result in a higher overall gain and a lower noise figure.

For an ideal circulator, no signal is going from its ports $\mathrm{C}$ to $\mathrm{B}$. Hence, both signal paths are completely separated from each other. One of the two amplifiers is a high power amplifier and the other one a low noise amplifier. Both amplifiers are designed and optimized to work in a standard 50-Ù-system. Both make use of so-called gain blocks, which are integrated circuits designed for a wide frequency range between $100 \mathrm{MHz}$ and $3 \mathrm{GHz}$ and need only a few additional elements for operation. In Figure 1 all elements with their values are given. With this circuit each amplifier path shows a reflection of less than $-11 \mathrm{~dB}$ and a gain of about $+9 \mathrm{~dB}$. The amplifiers are combined with circulators. These circulators can handle high power of $50 \mathrm{~W}$, whereas their mechanical dimensions are only of the size of $20 \times 20 \mathrm{~mm}^{2}$. Their insertion loss with $0.5 \mathrm{~dB}$ is sufficiently low, and the isolation has been measured to be -18 and $-15 \mathrm{~dB}$ at 2.4 and $2.5 \mathrm{GHz}$, respectively.

Figure 2 shows the $S$-parameter measurement results of the bidirectional amplifier using circulators. Hereby are $S_{21}$ and $S_{12}$ defined as the forward and reverse gains, respectively. $S_{11}$ and $S_{22}$ are the reflection coefficients at ports 1 and 2, respectively. All $S$-parameters are given as a magnitude in $\mathrm{dB}$.

As seen in Figure 2, all $S$-parameters show the same frequency characteristic. The forward and reverse gain dropped down to $\sim 5$ $\mathrm{dB}$ within the ISM band. The reflections at ports 1 and 2 are positive and show values of $+5 \mathrm{~dB}$ within the ISM band. Moreover, two significant resonance peaks are observed, one at 2.37 $\mathrm{GHz}$ and the other at $2.59 \mathrm{GHz}$. This indicates that this bidirectional amplifier is highly unstable. The reasons for this instability and a redesign of the circuit to overcome these problems are presented later.

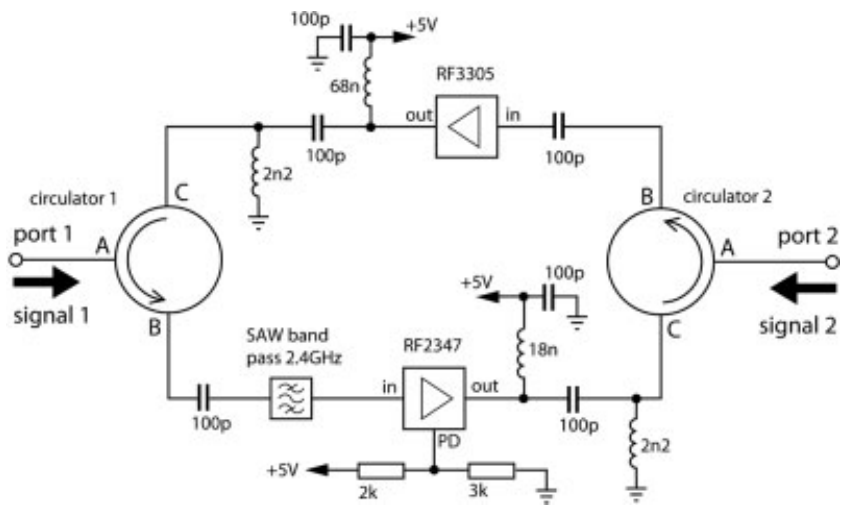

Figure 1 Schematic of the bidirectional amplifier realized with circulators 


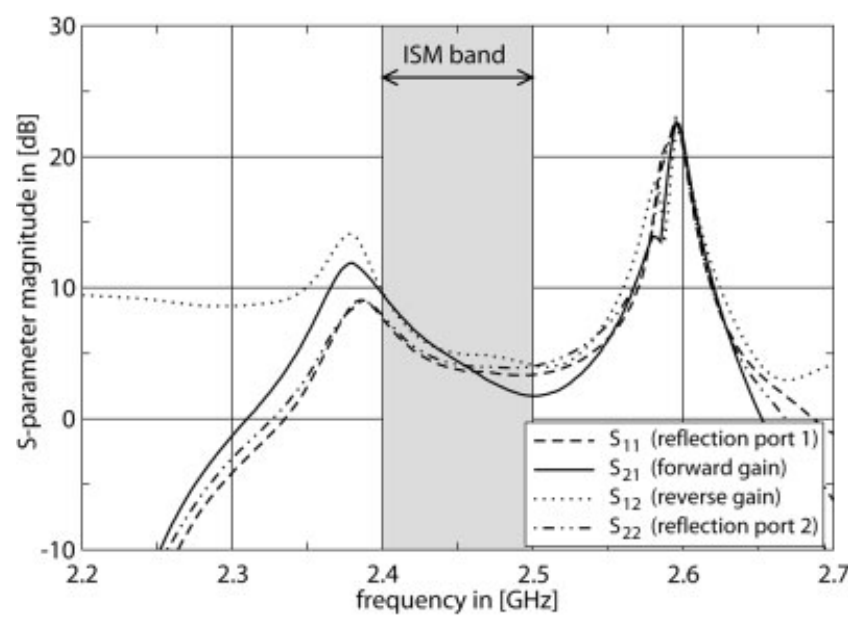

Figure 2 Measured $S$-parameters of the switchless bidirectional amplifier

\subsection{Problem Analysis}

An investigation in Section 2.1 observed that unstable behavior of the amplifier reveals that the problem of resonance and reflection can be solved independently.

2.2.1. Resonance. The reason for the two observed resonances lies in the nonideal behavior of the circulators and, in particular, in the layout of the amplifier. In Figure 3 the layout of the bidirectional amplifier is depicted, whereas the signal path causing the resonances is marked with a dashed line. Because of the limited isolation of the circulators, the signal path via the two amplifiers and circulators forms a ring resonator. The length of the signal path defines the circumference of the ring resonator and, therefore, the resonance frequencies. As depicted in Figure 3, the circumference was measured to be $L=2 \times 27 \mathrm{~mm}+2 \times 35 \mathrm{~mm}=124 \mathrm{~mm}$. This results in a minimum resonance frequency, which can be determined as

$$
f_{\text {res }}=\frac{c_{0}}{n \cdot \lambda}=\frac{c_{0}}{L}=2.42 \mathrm{GHz} \quad \text { with } n=1
$$

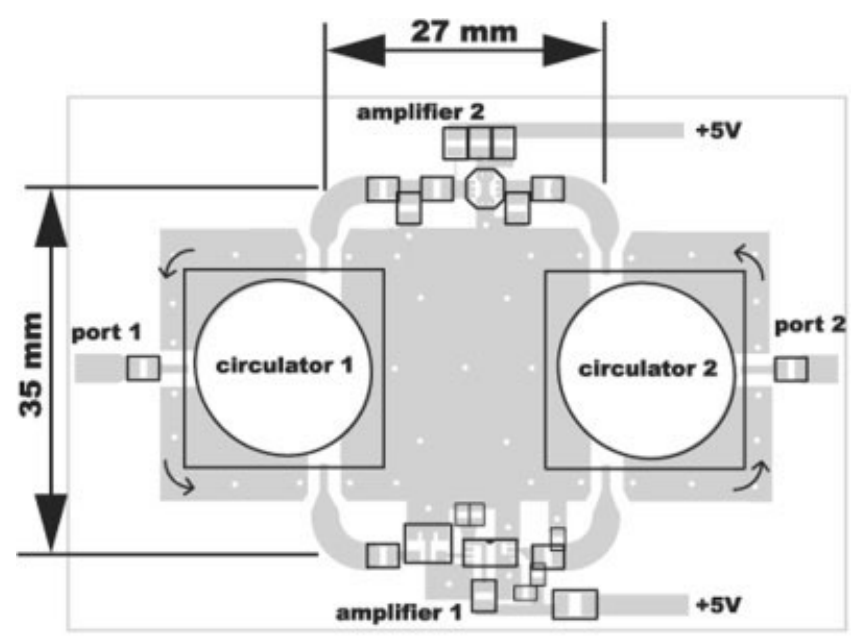

Figure 3 Layout of the switchless bidirectional amplifier with marked signal path causing resonances
Resonance frequencies of higher modes $(n=2,3, \ldots)$ are not of interest, since the used amplifiers do not work at these frequencies. The measured two resonances are close to the estimated one. The difference between the measurement and the result from Eq. (1) is due to parasitic effects of the layout which have not been taken into account in this estimation. To solve this problem, the layout has to be redesigned. This will be described in Section 2.3.

2.2.2. Reflection. The second problem is the observed reflection at ports 1 and 2 . For total reflection the reflection coefficient becomes 1 which corresponds to $0 \mathrm{~dB}$. However, the measured reflection coefficients $S_{11}$ and $S_{22}$ are even larger than 1 which corresponds to positive $\mathrm{dB}$-values. This means that the reflected signal is stronger than the ingoing signal. To illustrate the problem, the block diagram of the bidirectional amplifier together with the signal flow cause reflection $S_{11}$. For the sake of simplicity, second order effects have been neglected. Second order effects are e.g. reflections at the ports of amplifiers 1 and 2 and circulators 1 and 2.

Following the signal flow in Figure 4 the reflection coefficient $S_{11}$ can be calculated as

$$
S_{11}=\left.\frac{b}{a}\right|_{\text {port 2 ter min ated }}=G_{1} G_{2} I_{2}
$$

whereas $G_{1}, G_{2}$, and $I_{2}$ are the gain of amplifier 1 , the gain of amplifier 2, and the isolation of circulator 2, respectively. Regarding Eq. (2), one mean to reduce the reflection is to reduce the gain of the amplifiers. However, reducing the gain does not comply with the requirements of wireless communication systems. Hence, for an as low as possible reflection, the isolation of the circulators must be as good as possible. When all values are given in $\mathrm{dB}$, the reflection $S_{11}$ is the sum of $G_{1}, G_{2}$, and $I_{2}$ in $\mathrm{dB}$. Interestingly, only the isolation of circulator 2 affects the reflection $S_{11}$. Similarly, the reflection $S_{22}$ is affected only by the isolation of circulator 1 . In Section 2.1 the gain values for amplifiers 1 and 2 were measured to be $9 \mathrm{~dB}$ each. Together with a permitted maximum reflection of $-10 \mathrm{~dB}$, the isolation must show a value of less than $-28 \mathrm{~dB}$ within the frequency band of interest. As previously mentioned, the circulators show an insufficient isolation of about $-15 \mathrm{~dB}$.

\subsection{Redesign of the Amplifier}

To avoid resonances due to nonideal isolation of the circulators and the length of the signal path, a new layout has been designed. Thereby, the circumference of the signal path has been reduced to a size of $78 \mathrm{~mm}$. This corresponds to a minimum resonance

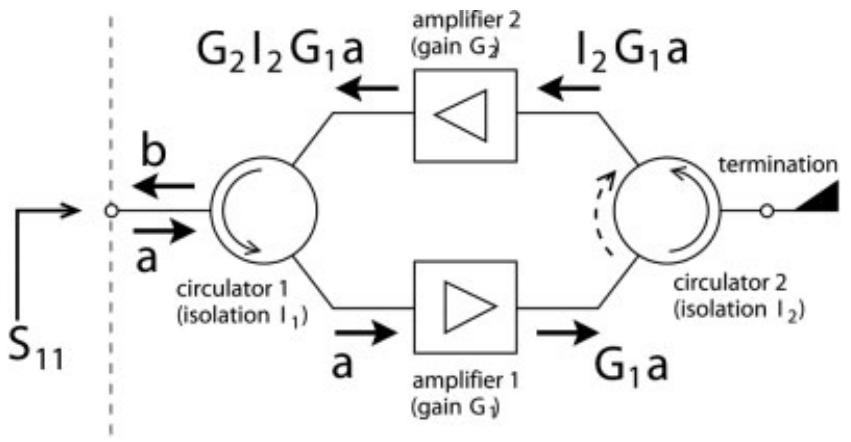

Figure 4 Block diagram with signals of the switchless bidirectional amplifier 


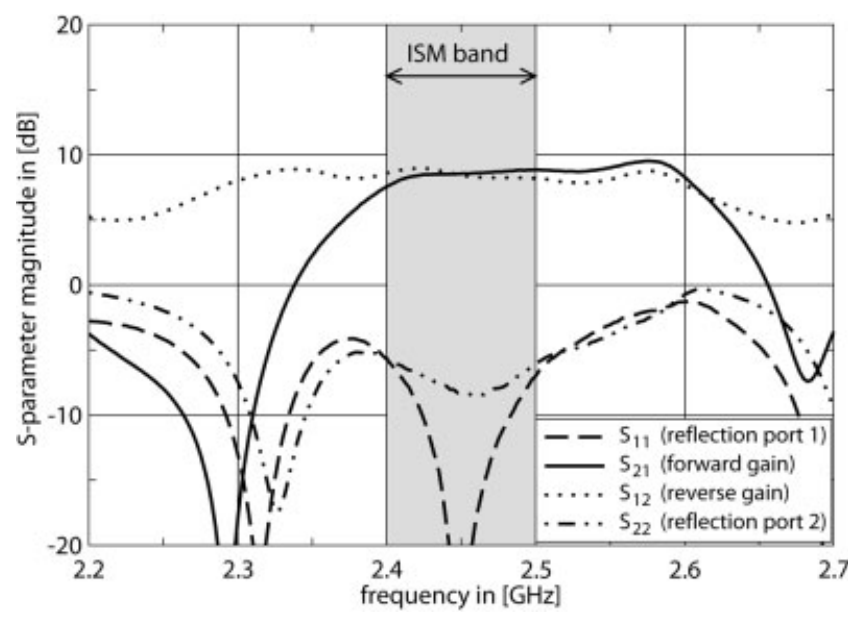

Figure 5 Measured $S$-parameters of the redesigned bidirectional amplifier

frequency of $3.85 \mathrm{GHz}$. The used SAW band pass filter blocks this frequency and no resonances have been observed.

Within this work it was possible to improve the isolation within the frequency band of interest by mounting small magnets onto both circulators. Important for the magnet is its magnetic strength and position on the circulator. As known, microwave circulators are devices which use a biased ferrite as a resonating nonreciprocal element [9]. By changing the biasing magnetic field, the optimum performance can be tuned to a small extent.

Figure 5 shows the measured $S$-parameters of the redesigned switchless bidirectional amplifier. Both gains are $8 \mathrm{~dB}$ and no resonance is observed. The bandpass characteristic of $S_{21}$ can be attributed to the SAW filter connected to amplifier 1 . The reflections of ports 1 and 2 show values of less than $-6 \mathrm{~dB}$.

\section{CONCLUSION}

With means of circulators a switchless bidirectional amplifier working at $2.4 \mathrm{GHz}$ has been presented. The advantage of this amplifier is that two signals can be amplified simultanously. Data loss due to delay times like with a switched system is avoided. Moreover, each signal path can be optimized according to its specific task. Another advantage is the low insertion loss and high power handling capacity of circulators. Both, noise and intermodulation can be kept to a minimum. Furthermore, the amplifier has been fabricated on a single PCB with the size of only $50 \times 35$ $\mathrm{mm}^{2}$.

For proper operation, the layout of the circuit is of importance. A smallest possible size of the layout shifts possible resonances to higher frequencies than the frequency band of interest. Thus, with means of a band pass filter those resonances can be easily suppressed. Furthermore, the isolation of the used circulators must be as good as possible. The major effect of a poor isolation is a high reflection at the ports of the bidirectional amplifier. In extreme cases, as observed, the reflected signal is even of higher amplitude than the ingoing signal.

The disadvantage of the bidirectional amplifier using circulators is its price. Circulators are expensive, even when bought in large quantities.

\section{ACKNOWLEDGMENT}

The authors would like to thank the Scientific and Technical Research Council of Turkey (TUBITAK), for its financial support of the project.

\section{REFERENCES}

1. Antennafier 2400 CAE Series, RF Linx Corp., 2005.

2. WAF2400-1000 bi-directional amplifier, Winncom Technologies Corp., 2005

3. J.W. Archer, R.A. Batchelor, and O. Sevimli, Millimetre-wave Bidirectional Amplifiers, Topical Symp Millimet Waves (1997), 19-22.

4. J.M. Yang, R. Lai, Y.H. Chung, M. Nishimoto, M. Battung, W. Lee, and R. Kagiwada, Compact Ka-band Bi-directional amplifier for low-cost electronic scanning array antenna, IEEE J Solid-State Circuits 39 (2004), 1716-1719.

5. F. Denoth and B. Pellegrini, A transitorized directional coupler, IEEE Trans Circuit Theory 15 (1968), 177-184.

6. T.-H. Ho and S.-J. Chung, Compact Rf front-end configuration for short-range communication, Electron Lett 40 (2004), 314-315.

7. O.P. Leisten and R.J. Collier, Distributed amplifiers as duplexer/low crosstalk bidirectional elements in S-band, Electron Lett 24 (1988), 264-265.

8. T. Tsukii, S.G. Houng, and M.J. Schindler, Wideband bidirectional MMIC amplifiers for new generation T/R module, IEEE MTT-S Int Microwave Symp Dig, Boston, MA (1990), 907-910.

9. J. Helszajn, Waveguide junction circulators: Theory and practice, Wiley: New York, NY, 1998.

(C) 2007 Wiley Periodicals, Inc.

\section{CROSS-COUPLED HILBERT SPIRAL RESONATOR FOR BANDPASS FILTER DESIGN}

\author{
Ji-Chyun Liu, ${ }^{1}$ Po-Chiang Lu, ${ }^{1}$ Chi-Hsun Chien, ${ }^{1}$ \\ Jun-Mao Chang, ${ }^{1}$ Jong-Yih Chen, ${ }^{2}$ and Horng-Jyh Lin ${ }^{3}$ \\ ${ }^{1}$ Department of Electrical Engineering, Ching Yun University, Chung- \\ Li 320, Taiwan, Republic of China \\ 2 Department of Computer Science and Information Engineering, \\ Vanung University, Chung-Li, Taiwan, Republic of China \\ ${ }^{3}$ Department of Electronic Engineering, Vanung University, Chung-Li, \\ Taiwan, Republic of China
}

\section{Received 27 December 2006}

ABSTRACT: The modified cross-coupled spiral resonators with Hilbert configuration are introduced for a large coupling coefficient in this article. For comparison, the conventional cross-coupled spiral resonators are fabricated and measured herein. To obtain low insertion loss $(-0.27 \mathrm{~dB})$, high out-of-band rejection level $(-19.5 \mathrm{~dB})$ and wider $F B W(50.9 \%)$, the filter of simplified CCSR-H is proposed. Results included surface current distribution and frequency responses are presented and discussed. (C) 2007 Wiley Periodicals, Inc. Microwave Opt Technol Lett 49: 1890-1894, 2007; Published online in Wiley InterScience (www.interscience.wiley.com). DOI 10.1002/mop.22630

Key words: Hilbert; spiral resonator; cross coupled

\section{INTRODUCTION}

The attractive features of the spiral resonator are its compact size, low cost, high $\mathrm{Q}$, and low radiation loss. This character usually is applied to realize band-pass filters in microstrip

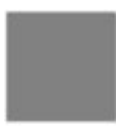

(a)

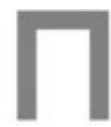

(b)

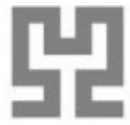

(c)

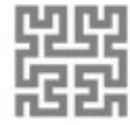

(d)

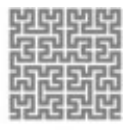

(e)
Figure 1 Hilbert iteration configurations (a) original, (b) first iteration, (c) second iteration, (d) third iteration, and (e) fourth iteration 